\title{
Soft materials based on colloidal self-assembly in
}

\section{ionic liquids}

\author{
Kazuhide Ueno*
}

Department of Chemistry and Biotechnology, Yokohama National University, 79-5 Tokiwadai, Hodogaya-ku, Yokohama 240-8501, Japan

CORRESPONDING AUTHOR FOOTNOTE: To whom correspondence should be addressed.

Telephone/Fax: +81-45-339-3951. E-mail: ueno-kazuhide-rc@ynu.ac.jp 


\begin{abstract}
Ionic liquids (ILs) have attracted much attention as dispersion media for colloidal systems alternatives to organic solvents and electrolyte solutions. Although colloidal stability is an essential factor for determining the properties and performance of colloidal systems combined with ILs, detailed mechanisms for the colloidal stabilization have not yet been studied. In the first part of this paper, we highlight our fundamental studies on the colloidal stability. Three different repulsive forces-electrostatic, solvation, and steric - are examined for their effectiveness to stabilize colloidal particles in ILs. In the second part, we provide an overview of our recent studies on colloidal soft materials in the presence of ILs. On the basis of suspended states of silica colloid particles, two different soft materials - colloidal gel and colloidal glass — were prepared in ILs. Their functional properties, including ionic transport, rheological properties, and optical properties, are discussed in relation to the microstructures of the colloidal materials.
\end{abstract}

KEYWORDS: ionic liquids, colloidal stability, colloidal gel, colloidal glass 


\section{INTRODUCTION}

Gel materials containing an ionic liquid (IL) have received much attention in a variety of applications, because they can be handled as solid materials with inherent, IL-derived, unique properties, not found in molecular liquids. ${ }^{1,2}$ For instance, the properties of high thermal stability, high ionic conductivity, and wide electrochemical potential window have been exploited in an IL-based solid-state electrolyte for electrochemical devices (e.g., solid-state secondary batteries, fuel cells, solar cells, field effect transistors, and ionic polymer actuators). ${ }^{3,4}$ High selective solubility of certain compounds (especially $\mathrm{CO}_{2}$ ) and very low vapor pressure are considered attractive characteristics of ILs for membrane separation technology; there have been several reports on IL-based separation membranes in this area of research. ${ }^{5-7}$

Introduction of solid-like mechanical properties into ILs is generally achieved by forming organic or inorganic three-dimensional networks in ILs. Polymer gels containing ILs can be prepared by in-situ polymerization of monomers and cross-linkers ${ }^{8}$ or by self-assembly of ABA tri-block copolymers ${ }^{9}$, where the A and B segments are insoluble and soluble in the ILs, respectively. Certain polymers that undergo micro-phase separation can also be converted into self-standing polymeric membranes in the presence of ILs. ${ }^{10-12}$ Inorganic networks have been constructed by in-situ sol-gel reactions of metal alkoxides in ILs, where porous skeletons of the resulting metal oxide monoliths (e.g., silica) are impregnated with the ILs. ${ }^{13}$ Another facile procedure for obtaining gel-like materials of ILs is to employ colloidal assembly of nanoparticles (NPs) in ILs, ${ }^{14}$ and we highlight recent studies on this class of soft materials in this paper.

The fluidity of a colloidal suspension may be entirely lost when the particle concentration increases to a certain critical concentration (known as jamming transition). ${ }^{15}$ There are two categories of solid-like soft materials consisting of colloidal suspensions, i.e. colloidal gel and colloidal glass, based on the internal structure of the particle assembly (Figure 1). ${ }^{16}$ A colloidal gel is generally formed with colloidally unstable particles in a medium where interparticle attractive force is dominant. The particles aggregate into a fractal-like network, which percolates through the volume of the suspension, even at relatively small particle concentrations. On the other hand, colloidal glass is often formed with colloidally stable, 
repulsive particles. In suspensions with concentrations above the critical particle concentration, the translational motion of each particle is inhibited by crowded neighboring particles (cage effect). ${ }^{17}$ Therefore, the entire system gets trapped into a metastable frozen state, and shows a solid-like response.

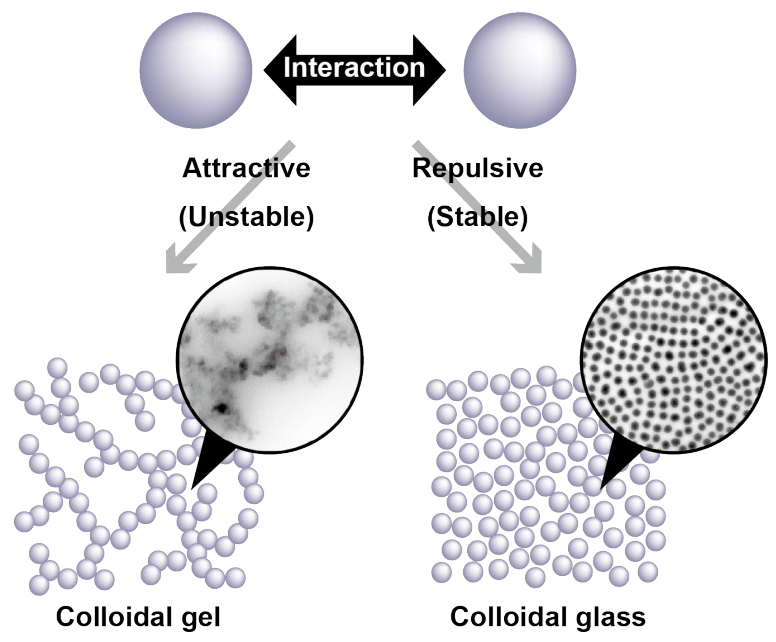

Figure 1. Schematic illustration for colloidal gel and colloidal glass, formed with unstable and stable nanoparticles (NPs), respectively. Representative TEM images of the soft colloidal materials formed in ILs: (left) $5 \mathrm{wt} \%$ fumed silica NPs $(R=6 \mathrm{~nm})$ in $\left[\mathrm{C}_{2} \mathrm{mim}\right]\left[\mathrm{NTf}_{2}\right]$ and (right) $16.7 \mathrm{wt} \%$ poly(methyl methacrylate) (PMMA)-grafted silica NPs $\left(M_{\mathrm{n}}=53 \mathrm{kDa}\right)$ in $\left[\mathrm{C}_{2} \mathrm{mim}\right]\left[\mathrm{NTf}_{2}\right]$.

Although the formation of the soft materials is governed primarily by the colloidal interactions as mentioned above, the details of interparticle attractive and repulsive forces in ILs is not yet understood. In connection with the applications of colloidal systems having ILs, there has been several works on the synthesis of metal and semiconductor NPs, ${ }^{18}$ on the catalytic processes using noble metal NPs, ${ }^{19}$ and on the preparations of functional NP-IL composites ${ }^{20,21}$; some of the works have reported unusual enhancements of colloidal stability of NPs in ILs in the absence of additional stabilizers. Hence, understanding of the colloidal stability of NPs in ILs is also an important research topic. In this respect, our group has been investigating silica NPs as a model, keeping the following queries in mind: (1) how the NPs get stabilized in ILs? (2) what type of repulsions is actually effective to stabilize the NPs in ILs? (3) can we prepare the above two colloid-based soft materials in the presence of ILs? In the first part of this paper, we will report on our fundamental studies on the interparticle interactions acting in ILs. In the second part, we will discuss the functional properties of the colloidal gels and colloidal glasses containing ILs. 


\section{INTERPARTICLE FORCES IN IONIC LIQUIDS}

Colloidal stability of NPs in suspensions is determined by the interplay of attractive/repulsive forces; the NPs can be kinetically stabilized when the repulsive forces are much stronger than the attractive forces. Van der Waals force is a universal and predominant attractive force acting in the colloidal system. ${ }^{22}$ Unlike the molecules in molecular systems, colloidal NPs are considered as a molecular assembly of a large number of molecules; for this reason, van der Waals potential energy ( $\left.V_{\mathrm{vdw}}\right)$ brings forth a strong and long-range attraction, proportional to the radius of the NPs $(R)$ and inversely proportional to the interparticle distance $(D)$. This is strong enough to form the colloidal aggregates, if no effective repulsions are present. In addition, $V_{\mathrm{vdw}}$ is a function of refractive index of not only the NPs but also the medium: The closer these two refractive indices are, the weaker the $V_{\mathrm{vdw}}$ is. The matching of refractive indices of the silica and the used IL allowed minimization of the $V_{\mathrm{vdw}}$ in the colloidal systems studied here.

Electrostatic force is a repulsive force derived from the electrical double layer (EDL) of a charged NP. ${ }^{23}$ Most importantly, electrostatic potential energy $\left(V_{\text {ele }}\right)$ strongly depends on the ionic concentration in the medium. Thickness of the EDL decreases as the ionic concentration increases, and the $V_{\text {ele }}$ becomes weaker in a concentrated salt solution. Therefore, it is easy to imagine that $V_{\text {ele }}$ would not effectively act in an IL, where there is a severe charge screening due to extremely high ionic concentration of the IL.
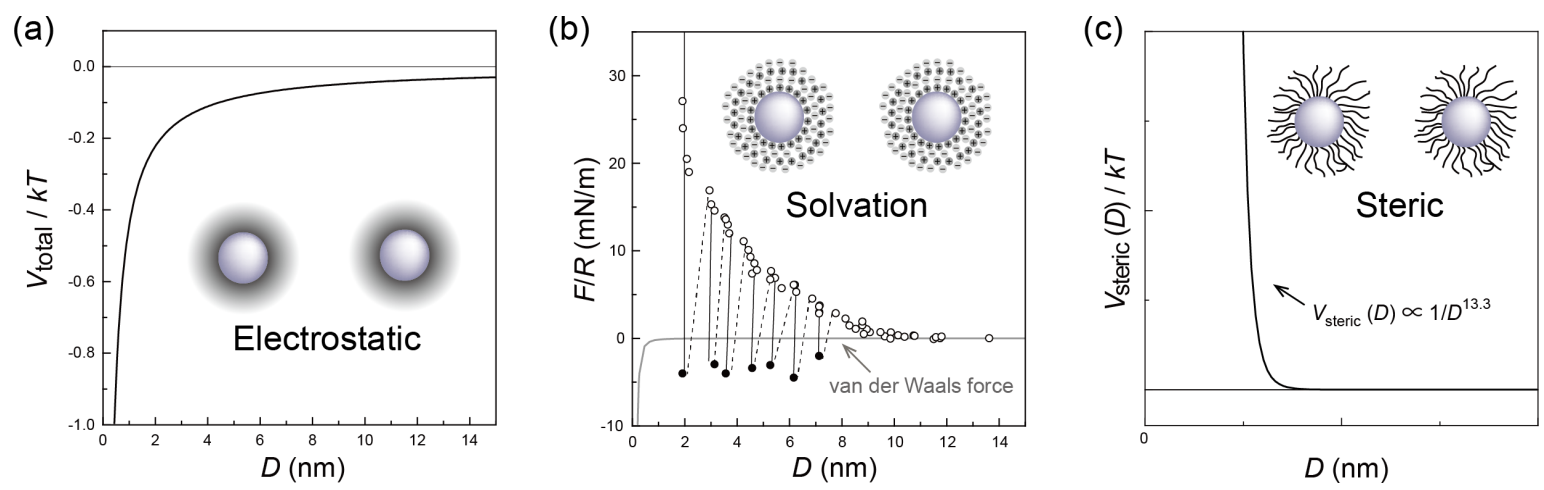

Figure 2. (a) Total potential energy $\left(V_{\text {total }} / k T\right)$, calculated for negatively charged silica NPs $(R=60 \mathrm{~nm})$ in $\left[\mathrm{C}_{2} \mathrm{mim}\right]\left[\mathrm{NTf}_{2}\right]$. (b) Normal force scaled with the surface radius of curvature $(F / R)$ as a function of the surface separation $D$ between silica surfaces in $\left[\mathrm{C}_{4} \mathrm{mim}\right]\left[\mathrm{NTf}_{2}\right]$; the gray solid line represents the calculated 
van der Waals force. (c) Predicted steric repulsive force between PMMA-grafted silica NPs $\left(M_{\mathrm{n}}=132\right.$ $\mathrm{kDa})$ in $\left[\mathrm{C}_{2} \mathrm{mim}\right]\left[\mathrm{NTf}_{2}\right]$.

Considering van der Waals attraction and electrostatic repulsion, colloidal stability can be simply described on the basis of Derjaguin-Landau-Verwey-Overbeek (DLVO) theory, in which $V_{\text {total }}=V_{\mathrm{vdw}}+$ $V_{\text {ele. }}{ }^{23}$ Even considering silica-IL systems with a reliable surface charge potential of $-38 \mathrm{mV}, V_{\text {total }}$ only shows an attractive interaction between the silica particles in the whole separations $(D)$, as can be seen in Figure 2a. Although different ionic liquids with reasonable surface charge potentials ranging from -38 $\mathrm{mV}$ to $-52 \mathrm{mV}$ were also examined in the same manner, all the interparticle interaction profiles indicated similar attractive interactions at all the separations. This prediction, using DLVO theory, can be verified through the dynamic light scattering (DLS) experiments, showing aggregates of silica NPs $(R=60 \mathrm{~nm})$ suspended in the ILs. ${ }^{24}$

To gain information on the presence or absence of repulsive interactions between silica NPs, the fractal-like aggregation was further investigated on the basis of two different models of cluster-cluster aggregation, diffusion-limited aggregation (DLA), and reaction-limited aggregation (RLA). ${ }^{25}$ Every particle collision causes an aggregation in DLA; however, the aggregates are formed only when the particle collision overcomes a moderate energy barrier comparable to or larger than $k T$ in RLA. Therefore, the fractal aggregation has a loosely packed structure and a denser structure in DLA and RLA, respectively. The aggregation was formed through RLA in an IL, $\left[\mathrm{C}_{2} \mathrm{mim}\right]\left[\mathrm{NTf}_{2}\right]^{26}$, while DLA was reported for the same silica NPs suspended in mineral oil. ${ }^{27}$ This implies that another repulsive force is present between the silica NPs; the non-electrostatic repulsion, however, was not strong enough to stabilize the silica NPs in the case of $\left[\mathrm{C}_{2} \mathrm{mim}\right]\left[\mathrm{NTf}_{2}\right]$. It was found that the same silica NPs are well stabilized in BF 4 -based ILs by the non-electrostatic repulsion. ${ }^{28}$

Figure 2b shows actual surface force $(F / R)$ between silica surfaces in an IL, which was measured using a surface force apparatus. ${ }^{29}$ The surface force measurement revealed the presence of nonelectrostatic repulsive force. The repulsion has an oscillatory profile, characterized as the solvation force resulting from the formation of a layered structure in the vicinity of solid surfaces, when liquid molecules 
are confined to nanoscale. ${ }^{30}$ Similar layer-by-layer structures of cations and anions of ILs on charged surfaces have also been suggested in AFM force measurements, high-energy X-ray reflectivity measurements, and molecular dynamics simulations. ${ }^{31}$ The intrinsic structure-forming properties of ILs in bulks would lead to the formation of remarkably well-defined layered structures at charged surfaces. Although many ILs were found to show the solvation force ${ }^{32}$ and it can be an efficient repulsion for stabilizing NPs in the highly ionic media, colloidal stabilization of NPs by the IL-based solvation force was found in the limited cases. In addition to the above-mentioned $\mathrm{BF}_{4}$-based ILs, the unusual stabilization by the IL-based solvation force was reported for silica NPs suspension in a protic IL, ethyl ammonium nitrate. ${ }^{33}$ Recent study has shown that surface-ion hydrogen bonding leads to a strong solvation force enabling the colloidal stabilization in this type of ILs. ${ }^{34}$ It was also recently reported that metal and semiconductor NPs can be stabilized by a layer of surface-bound ions in inorganic ILs. ${ }^{35}$

The steric repulsion, derived from excluded volume effect of surface functionalized groups on NPs, was examined using poly(methyl methacrylate)-grafted silica NPs (PMMA-g-NPs) in ILs. In a good solvent, the penetration of the grafted polymers gives rise to a repulsion between polymer-grafted NPs. ${ }^{36}$ The observation by DLS and TEM proved that PMMA-g-NPs, where PMMA is soluble, are suspended as primary particles without aggregation in the $\mathrm{ILs}^{24}$ Rheological measurements for concentrated suspensions of PMMA-g-NPs, having $M_{\mathrm{n}}=132 \mathrm{kDa}$, have also shown that PMMA- $g$-NPs have a soft repulsive potential energy $\left(V_{\text {steric }}(D)\right)$ proportional to $D^{-13.3}$ in $\left[\mathrm{C}_{2} \operatorname{mim}\right]\left[\mathrm{NTf}_{2}\right]^{37}$, which is due to deformable outer shell of the grafted PMMA swollen with $\left[\mathrm{C}_{2} \mathrm{mim}\right]\left[\mathrm{NTf}_{2}\right]$ (Figure 2c).

Colloidal stability of PMMA-g-NPs was also studied in various ILs, since the steric interaction is dominated by the solubility of the grafted polymer in the medium. ${ }^{38} V_{\text {steric }}(D)$ is attractive in a poor solvent for the grafted polymer. In other words, the DLS measurements of PMMA-g-NPs enabled us to evaluate the details of solubility of the PMMA in the ILs. Figure 3 shows hydrodynamic radius $\left(R_{\mathrm{h}}\right)$ of PMMA- $g$ NPs in the alkylimidazolium ILs having different alkyl chain lengths and anionic structures. The PMMA$g$-NPs were aggregated in all the $\mathrm{BF}_{4}$-based ILs, defined as $R_{\mathrm{h}}=60 \mathrm{~nm}$ in Figure 3, because of the insolubility of the PMMA in these ILs. The $R_{\mathrm{h}}$ became larger in the ILs with more hydrophobic anions, 
in the following order: $[\mathrm{TfO}]<\mathrm{PF}_{6}<\left[\mathrm{NTf}_{2}\right]$-based ILs. When the anionic structure is the same, $R_{\mathrm{h}}$ has a relatively good relationship with the nonpolar solubility parameter $\left(\delta_{\mathrm{NP}}\right)$, proposed by Coutinho et al. ${ }^{39}$, and becomes maximum at $\delta_{\mathrm{NP}}=\sim 22 \mathrm{MPa}^{0.5}$. The above study suggests that the whole balance of the nonpolar character between the cations and anions is associated with the solubility of PMMA. The effect of nonpolar property of the anion is more predominant over the effect of solubility; the nonpolar property of the cation also modifies the overall affinity towards PMMA.

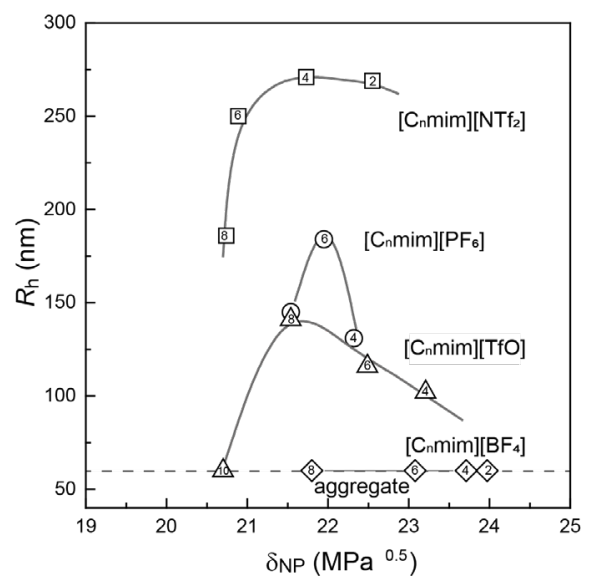

Figure 3. Hydrodynamic radius $\left(R_{\mathrm{h}}\right)$ of PMMA- $g$-NPs $\left(M_{\mathrm{n}}=180 \mathrm{kDa}\right)$ in $\left[\mathrm{C}_{\mathrm{n}} \mathrm{mim}\right]$-based ILs with various anionic structures at $25^{\circ} \mathrm{C}$, plotted as a function of nonpolar solubility parameter $\left(\delta_{\mathrm{NP}}\right)$ of ILs reported by Coutinho et al. ${ }^{39}$

Through the fundamental studies on the interparticle interactions in ILs, the following answers were obtained to the original question about the colloidal stability of NPs in ILs. The electrostatic repulsion is found to be inefficient due to the concentrated ionic atmosphere in ILs. Alternatively, the solvation and steric forces have a potential to stabilize NPs in ILs. Therefore, IL-based steric and IL-based solvation forces would rationalize the reported experimental findings for pronounced colloidal stability in ILs, in absence of any stabilizer.

\section{COLLOIDAL GELS AND COLLOIDAL GLASSES IN IONIC LIQUIDS}

The IL-based colloidal gels can be readily prepared by mixing, for example, unstable, fumed silica NPs with ILs. The poorly stable silica NPs form interconnecting networks with a fractal structure in the 
ILs, as shown in Figure 1. Gelation of $\left[\mathrm{C}_{2} \mathrm{mim}\right]\left[\mathrm{NTf}_{2}\right]$ could be achieved with the addition of only $\geq 2$ wt $\%$ ( $\geq 1.5 \mathrm{vol} \%$ ) of fumed silica NPs with an average radius $R$ of $6 \mathrm{~nm} .{ }^{26}$ The system showed an evidently higher elastic modulus $\left(G^{\prime}\right)$, compared to viscous modulus $\left(G^{\prime \prime}\right)$, through the rheological measurement by dynamic frequency-sweeps, thereby indicating a typical, soft-solid-like response. On the other hand, a larger particle concentration was required for the IL-based colloidal glass. ${ }^{37}$ The particle concentration required for the quasi-solidification was $6 \mathrm{wt} \%$ for PMMA- $g$-NPs $\left(M_{\mathrm{n}}=132 \mathrm{kDa}\right)$. This corresponds to an effective volume fraction ( $\phi_{\text {eff }}$ ) of $\sim 70-74$ vol $\%$, including the silica core and the solvated shell of PMMA. The $\phi_{\text {eff }}$ value was almost independent of $M_{\mathrm{n}}$ of the grafted PMMA in PMMA-g-NPs, but it was higher than the volume fraction of random-close packing for a hard sphere system ( $\sim 64 \mathrm{vol} \%)$, owing to the soft repulsive potential of PMMA-g-NPs (i.e., the deformability of the PMMA shell). The colloidal crystal of PMMA-g-NPs in $\left[\mathrm{C}_{4} \mathrm{mim}\right]\left[\mathrm{NTf}_{2}\right]$ was prepared by Ohno et al. ${ }^{40}$ The volume fractions of PMMA-g-NPs for freezing and melting were reported to be 23.3 and 24.9 vol\%, respectively.

As the loosely bound network could have been disrupted under sufficient shear stress, the colloidal gel showed a reversible "shear thinning" behavior (Figure 4). ${ }^{28}$ The gel modulus $\left(G^{\prime}\right)$ increased with the particle concentrations, and reached $\sim 10^{6} \mathrm{~Pa}$ at $15 \mathrm{wt} \%$. The ionic structure of ILs was found to affect the formation of colloidal gels. Indeed, no gelation occurred with the same fumed silica NPs in $\mathrm{BF}_{4}$-based ILs, in which the silica NPs were stabilized by the IL-based solvation forces, as discussed in the former section. These stable suspensions exhibited an interesting "shear thickening" response (Figure 4). ${ }^{28} \mathrm{~A}$ sudden increase in shear viscosity can probably be attributed to a temporary aggregation of NPs, associated with collapsing of the surface solvation structure of the ions at an intermediate shear rate. Further dissociation of the aggregates is responsible for the decrease in viscosity at high shear rate. The colloidal glass of PMMA-g-NPs also exhibited shear thinning behavior and significant increase in the gel modulus with increase in the particle concentration. ${ }^{37}$ 


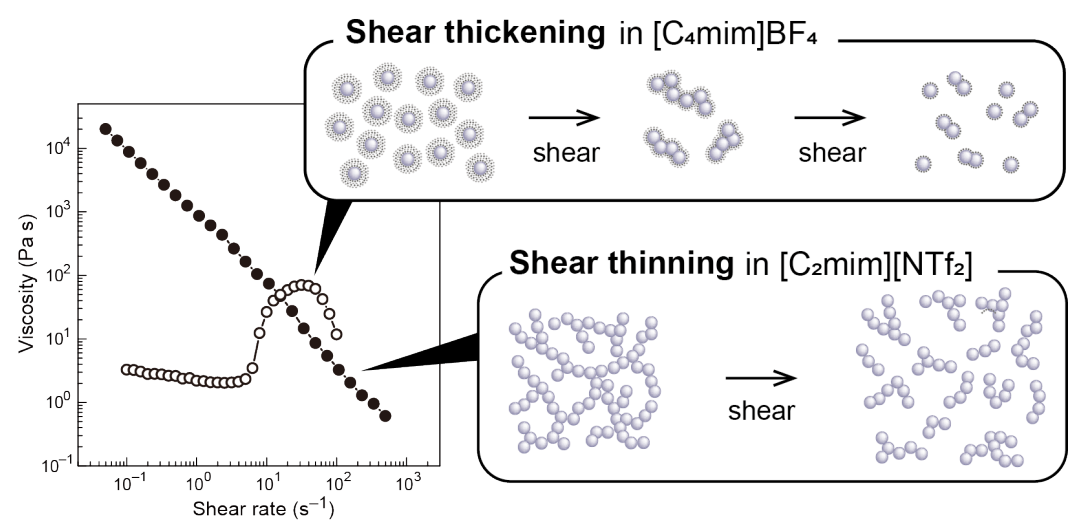

Figure 4. Shear rate dependence of viscosity for a colloidal gel (closed circle) of $5 \mathrm{wt} \%$ fumed silica NPs $(R=6 \mathrm{~nm})$ in $\left[\mathrm{C}_{2} \mathrm{mim}\right]\left[\mathrm{NTf}_{2}\right]$ and a stable suspension (open circle) of $15 \mathrm{wt} \%$ fumed silica NPs in $\left[\mathrm{C}_{4} \mathrm{mim}\right]\left[\mathrm{BF}_{4}\right]$, and schematic illustrations for the changes in the microstructure by shear.

One of the important properties of the IL-based soft materials is the ionic transport property. The addition of fumed silica NPs $(R=6 \mathrm{~nm})$ had little impact on the ionic conductivity of the colloidal gel with $\left[\mathrm{C}_{2} \mathrm{mim}\right]\left[\mathrm{NTf}_{2}\right] \cdot{ }^{26}$ Ionic conductivity was lower for the colloidal glass (Figure 5a). ${ }^{37}$ The ionic conduction was more inhibited by their interactions with PMMA in addition to the obstruction of the silica NPs. Diffusivity measurements, using pulsed field gradient (pfg-) NMR, have shown different interactions of ion-NPs in the colloidal gel and colloidal glass, where $D_{0}$ and $D_{\mathrm{g}}$ are self-diffusion coefficients of the ions in the parent IL and in the soft material, respectively. As seen in Figure 5b, the higher diffusivity ratio $\left(D_{\mathrm{g}} / D_{0}\right)$ of the cation for the colloidal glass suggests preferential interaction of [ $\left.\mathrm{NTf}_{2}\right]^{-}$anions with PMMA, while higher $D_{\mathrm{g}} / D_{0}$ of the anion for the colloidal gel is indicative of preferential interaction of the $\left[\mathrm{C}_{2} \mathrm{mim}\right]^{+}$cations with the silica surface. The importance of IL-based colloidal gels has been recognized in the recent studies on all-solid-state lithium ion batteries, where the colloidal gel was used as a solid electrolyte. ${ }^{41,42}$ The above results provide an insight into the role of interactions of ion-NPs in the developmental strategy for selective ion conductive materials or highly ion-conductive materials with high transference number of specific ions for energy storage devices. 


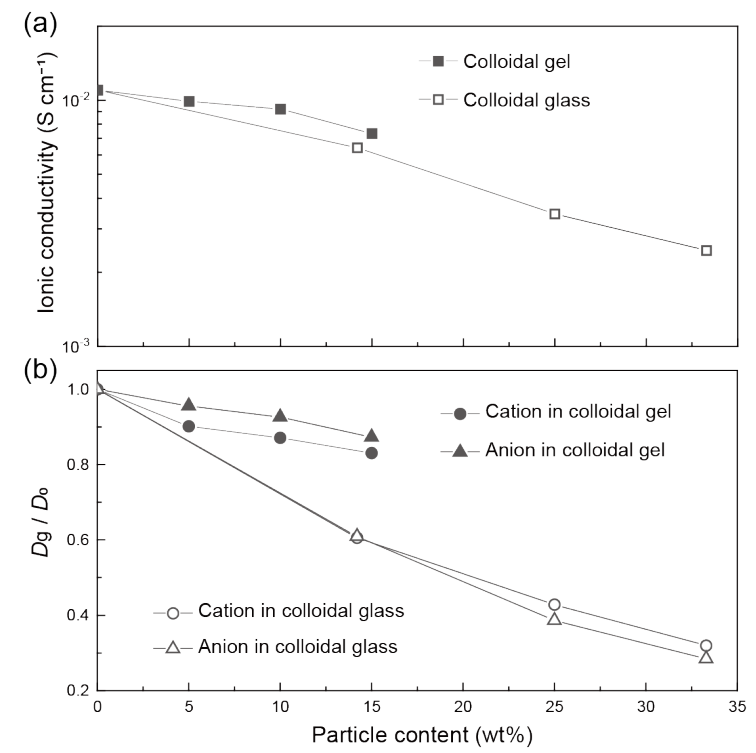

Figure 5. Dependence of (a) ionic conductivity and (b) diffusivity ratio $\left(D_{\mathrm{g}} / D_{0}\right)$ on the particle content for the colloidal gel of fumed silica NPs $(R=6 \mathrm{~nm})$ in $\left[\mathrm{C}_{2} \mathrm{mim}\right]\left[\mathrm{NTf}_{2}\right]$ and for the colloidal glass of PMMA- $g$-NPs $\left(M_{\mathrm{n}}=91 \mathrm{kDa}\right)$ in $\left[\mathrm{C}_{2} \mathrm{mim}\right]\left[\mathrm{NTf}_{2}\right]$ at $30{ }^{\circ} \mathrm{C}$.

Temperature sensitivity can be introduced into the grafted polymer for the polymer grafted NPs. In this way, the colloidal stability could be thermally controlled in the ILs. Poly(benzyl methacrylate) (PBnMA) is known to show lower critical-solution-temperature (LCST) phase separation in certain ILs at the phase separation temperature $\left(T_{\mathrm{c}}\right) .{ }^{43}$ The PBnMA-g-NPs can be sterically stabilized by the solvated PBnMA shell at a low temperature, whereas the PBnMA shell gets desolvated and the PBnMA-g-NPs forms aggregates above $T_{\mathrm{c}}$. The colloidal glass of PBnMA- $g$-NPs could be formed in $\left[\mathrm{C}_{2} \mathrm{mim}\right]\left[\mathrm{NTf}_{2}\right]$ in a similar way as PMMA-g-NPs, and it undergoes the transition of colloidal glass to colloidal gel, in response to temperature, as seen in TEM images in Figure 6. ${ }^{44}$ This transition was also characterized as a V-shaped rheological response. Both $G^{\prime}$ and $G^{\prime \prime}$ decreased with increasing temperature up to $95^{\circ} \mathrm{C}$, on account of the decrease in the particle volume fraction of the colloidal glass. However, the moduli increased again due to the formation of the interconnecting network of the aggregated PBnMA-g-NPs at higher temperatures. 


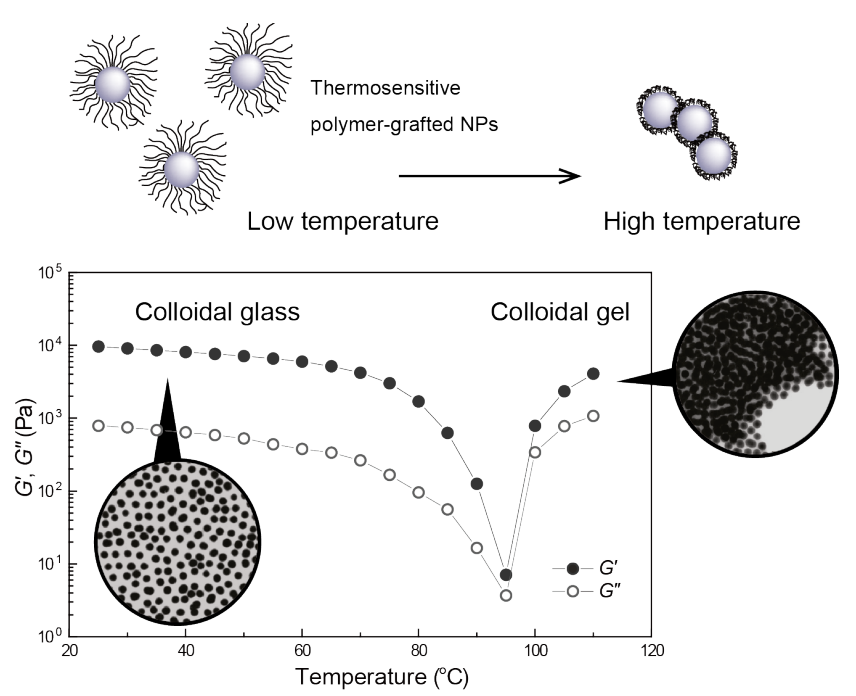

Figure 6. Schematic illustration for thermosensitive polymer grafted NPs. Temperature dependence of elastic modulus (closed circles) and viscous modulus (open circles) for the colloidal glass of $25.0 \mathrm{wt} \%$ PBnMA-g-NPs $\left(M_{\mathrm{n}}=101 \mathrm{kDa}\right)$ in $\left[\mathrm{C}_{2} \mathrm{mim}\right]\left[\mathrm{NTf}_{2}\right]$, and the pertinent TEM images.

It is also worth to note that the colloidal glass, consisting of relatively monodispersed polymer-grafted NPs, shows angle-independent structural colors from blue to green and red with decrease in the concentrations of the particles (Figures 7a and b). ${ }^{45}$ The theoretical work suggests that the angleindependent and non-brilliant structural color is due to the coherent scattering of visible light from the isotropic nanostructures with short-range order. ${ }^{46}$ TEM image and corresponding ring-shaped twodimensional (2D) Fourier power spectra corroborated that the short range ordered glassy structure led to the angle-independent structural colors (Figures $7 \mathbf{c}$ and $\mathbf{d}$ ). The slightly opaque color was due to the combination of an incoherent scattering from the amorphous structure in the long-range and a coherent scattering from the ordered structure in the short-range. 

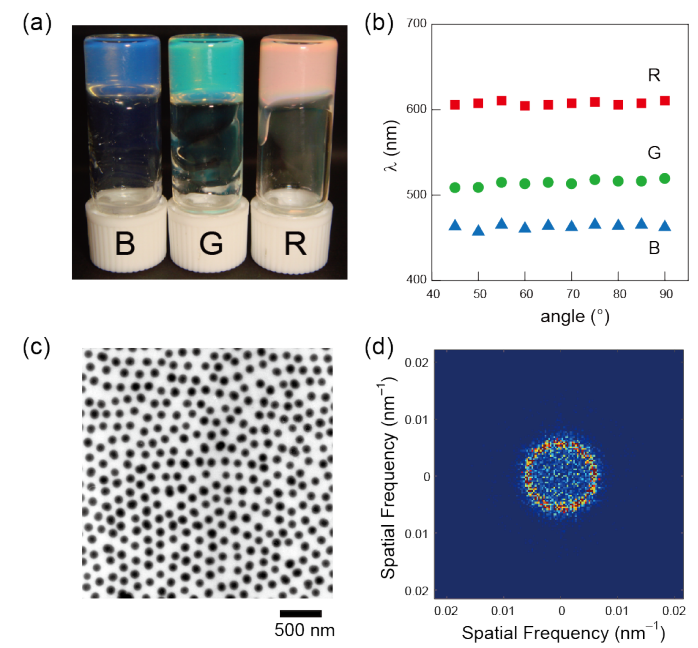

Figure 7. (a) Photograph of colloidal glass of PMMA-g-NPs $\left(M_{\mathrm{n}}=91 \mathrm{kDa}\right)$ in $\left[\mathrm{C}_{2} \mathrm{mim}\right]\left[\mathrm{NTf} \mathrm{N}_{2}\right]$ : (B) 33.3 $\mathrm{wt} \%$, (G) $25.0 \mathrm{wt} \%$, and (R) $14.2 \mathrm{wt} \%$. (b) Characteristic wavelength $(\lambda)$ measured as a function of the angle between the incident light and the supported glass substrate. (c) TEM image. (d) corresponding 2D Fourier power spectrum of the colloidal glass G.

Furthermore, the maximum wavelength of the reflection spectra $\left(\lambda_{\max }\right)$ can be empirically described as $\lambda_{\max } \approx 2 n_{\text {ave }} d$, where $d$ is the mean particle distance and $n_{\text {ave }}$ is the average refractive index for the colloidal glass. ${ }^{37}$ These results encourage us to tune the angle-independent structural color with temperature. For the colloidal glass of PBnMA-g-NPs, the structural color disappeared above $T_{\mathrm{c}}$ and the sample became an opaque gel. ${ }^{44}$ As seen in Figure 8a, the peak intensity of the reflection spectrum decreased with increasing temperature and no peak was observed at $89^{\circ} \mathrm{C}$. This is associated with the above-mentioned "colloidal glass to gel transition"; the short-range ordered structure in the glass state was transformed into a completely disordered structure in the gel state. Therefore, random light scattering of the aggregated PBnMA-g-NPs was dominant in the gel state. In a subsequent attempt, the colloidal glass was prepared with a di-block copolymer-grafted NPs, (PBnMA- $b$-PMMA)- $g$-NPs in order to mitigate the colloidal aggregation at temperatures above the $T_{\mathrm{c}}$ of PBnMA groups. ${ }^{47}$ In this case, the reflection spectrum shifted to a shorter wavelength at a higher temperature, without a significant decrease in the peak intensity (Figure 8b). The short-range-order structure could be retained even above $T_{\mathrm{c}}$, but the mean particle distance $d$ of the colloidal glass has decreased, because of the desolvation of PBnMA moiety. The peak shift was not large enough to tune the structural color in this attempt; therefore, factors such as size of the 
NPs and molecular weight of the grafted polymers need to be optimized to realize the tuning of the angleindependent structural color over the whole color range.

(a) PBnMA-grafted NPs

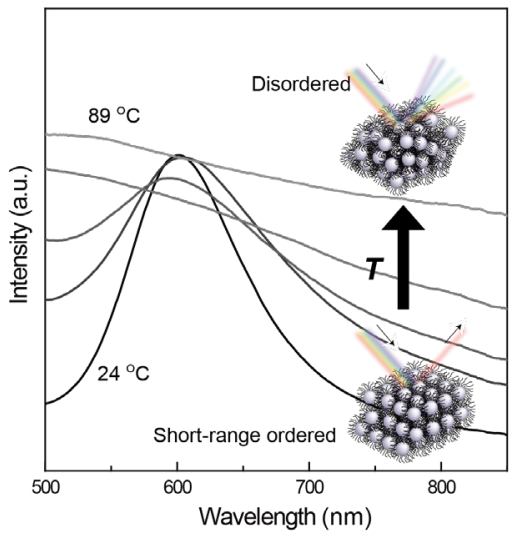

(b) PBnMA-b-PMMA-grafted NPs

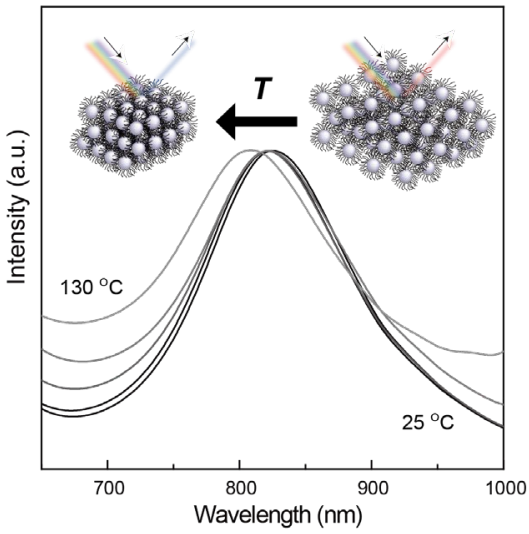

Figure 8. Temperature dependence of reflection spectra for the colloidal glass of (a) $25 \mathrm{wt} \% \mathrm{PBnMA}-g$ NPs $\left(M_{\mathrm{n}}=101 \mathrm{kDa}\right)$ and (b) $15 \mathrm{wt} \%$ (PBnMA-b-PMMA)- $g$-NPs $\left(M_{\mathrm{n}}=103 \mathrm{kDa}\right)$ in $\left[\mathrm{C}_{2} \operatorname{mim}\right]\left[\mathrm{NTf}_{2}\right]$.

\section{CONCLUSION}

Different fundamental studies on the colloidal stability of silica NPs in ILs are highlighted with a focus on three different repulsive interactions: electrostatic, solvation, and steric forces. A significant charge screening effect in the ILs makes the electrostatic force inefficient to separate the NPs. Alternatively, solvation and steric forces can act as valid repulsive forces to stabilize the NPs in the ILs. An IL-based solvation force was proposed to account for the experimental evidence for unusual colloidal stabilization in the ILs, in absence of any stabilizer. These stabilizer-free colloidal stabilizations should be more pronounced in ILs, where the amphiphilic property of ILs can promote encapsulation of colloidal particles. The intrinsic structure-forming property of ILs can also contribute to the formation of a well-defined layered structure on a colloidal surface (i.e., IL-based solvation force).

Two different soft materials were formed by the self-assembly of colloidal NPs in ILs, based on the colloidal stability. Colloidal gels were prepared using unstable silica NPs that formed an aggregated network in ILs, while colloidal glasses were formed by highly concentrated suspensions of sterically 
stabilized polymer-grafted NPs. For preparing IL-based solid electrolytes, the present colloidal approach may become a reasonable pathway to ensure liquid-like high ionic conductivity and solid-like high mechanical strength. Shear-induced fluidic properties may become an advantage in terms of facile processability. A variety of different rheological responses of the colloidal systems of ILs may also promise their use in a functional rheological fluid. Although silica NPs and PMMA/PBnMA were used as model core-particles and grafted polymers, respectively, many other functional colloidal particles and polymers are expected to be applicable to the IL-based colloidal gels and glasses.

\section{ACKNOWLEDGMENT:}

The author is deeply grateful to Prof. Masayoshi Watanabe, Dr. Takeshi Ueki, Prof. Kazue Kurihara, Prof. Masashi Mizukami, Dr. Motohiro Kasuya, and all colleagues for their collaborative works and fruitful discussions. This work was supported in part by JSPS Grant-in-Aid for JSPS Fellows Grant Number 08J02437. 


\section{REFERENCES}

1 Marr, P. C. \& Marr, A. C. Ionic liquid gel materials: applications in green and sustainable chemistry. Green Chem. 18, 105-128 (2016).

2 T. Carlin, R. \& Fuller, J. Ionic liquid-polymer gel catalytic membrane. Chem. Commun., 13451346 (1997).

3 MacFarlane, D. R., Forsyth, M., Howlett, P. C., Kar, M., Passerini, S., Pringle, J. M., Ohno, H., Watanabe, M., Yan, F., Zheng, W., Zhang, S. \& Zhang, J. Ionic liquids and their solid-state analogues as materials for energy generation and storage. Nat. Rev. Mater. 1, 15005 (2016).

4 Watanabe, M., Thomas, M. L., Zhang, S., Ueno, K., Yasuda, T. \& Dokko, K. Application of ionic liquids to energy storage and conversion materials and devices. Chem. Rev. 117, 7190-7239 (2017).

5 Cowan, M. G., Gin, D. L. \& Noble, R. D. Poly(ionic liquid)/ionic liquid ion-gels with high "free" ionic liquid content: platform membrane materials for $\mathrm{CO}_{2} /$ light gas separations. Acc. Chem. Res. 49, 724-732 (2016).

6 Gu, Y., Cussler, E. L. \& Lodge, T. P. ABA-triblock copolymer ion gels for $\mathrm{CO}_{2}$ separation applications. J. Membr. Sci. 423-424, 20-26 (2012).

7 Ranjbaran, F., Kamio, E. \& Matsuyama, H. Ion gel membrane with tunable inorganic/organic composite network for $\mathrm{CO}_{2}$ separation. Ind. Eng. Chem. Res. 56, 12763-12772 (2017).

8 Susan, M. A. B. H., Kaneko, T., Noda, A. \& Watanabe, M. Ion gels prepared by in situ radical polymerization of vinyl monomers in an ionic liquid and their characterization as polymer electrolytes. J. Am. Chem. Soc. 127, 4976-4983 (2005).

9 He, Y., Boswell, P. G., Bühlmann, P. \& Lodge, T. P. Ion gels by self-assembly of a triblock copolymer in an ionic liquid. J. Phys. Chem. B 111, 4645-4652 (2007).

10 Yeon, S.-H., Kim, K.-S., Choi, S., Cha, J.-H. \& Lee, H. Characterization of PVdF(HFP) gel electrolytes based on 1-(2-hydroxyethyl)-3-methyl imidazolium ionic liquids. J. Phys. Chem. B 109, 17928-17935 (2005).

11 Yasuda, T., Nakamura, S., Honda, Y., Kinugawa, K., Lee, S-Y. \& Watanabe, M. Effects of polymer structure on properties of sulfonated polyimide/protic ionic liquid composite membranes for nonhumidified fuel cell applications. ACS Appl. Mater. Interfaces 4, 1783-1790 (2012).

12 Ohno, H., Yoshizawa, M. \& Ogihara, W. A new type of polymer gel electrolyte: zwitterionic liquid/polar polymer mixture. Electrochim. Acta 48, 2079-2083 (2003).

13 Le Bideau, J., Viau, L. \& Vioux, A. Ionogels, ionic liquid based hybrid materials. Chem. Soc. Rev. 40, 907-925 (2011). 
14 Wang, P., Zakeeruddin, S. M., Comte, P., Exnar, I. \& Grätzel, M. Gelation of ionic liquid-based electrolytes with silica nanoparticles for quasi-solid-state dye-sensitized solar cells. J. Am. Chem. Soc. 125, 1166-1167 (2003).

15 Liu, A. J. \& Nagel, S. R. Jamming is not just cool any more. Nature 396, 21-22 (1998).

16 Stokes, J. R. \& Frith, W. J. Rheology of gelling and yielding soft matter systems. Soft Matter 4, 1133-1140 (2008).

17 Pusey, P. N. \& van Megen, W. Phase behaviour of concentrated suspensions of nearly hard colloidal spheres. Nature 320, 340 (1986).

18 Endres, F. Ionic liquids: solvents for the electrodeposition of metals and semiconductors. ChemPhysChem 3, 144-154 (2002).

19 Dupont, J., Fonseca, G. S., Umpierre, A. P., Fichtner, P. F. P. \& Teixeira, S. R. Transition-metal nanoparticles in imidazolium ionic liquids: recycable catalysts for biphasic hydrogenation reactions. J. Am. Chem. Soc. 124, 4228-4229 (2002).

20 Fukushima, T., Kosaka, A., Ishimura, Y., Yamamoto, T., Takigawa, T., Ishii, N. \& Aida, T. Molecular ordering of organic molten salts triggered by single-walled carbon nanotubes. Molecular ordering of organic molten salts triggered by single-walled carbon nanotubes. Science 300, 2072-2074 (2003).

21 Torimoto, T., Tsuda, T., Okazaki, K.-i. \& Kuwabata, S. New frontiers in materials science opened by ionic liquids. Adv. Mater. 22, 1196-1221 (2010).

22 Israelachvili, J. N. in Intermolecular and Surface Forces (Third Edition) 253-289 (Academic Press, 2011).

23 Israelachvili, J. N. in Intermolecular and Surface Forces (Third Edition) 291-340 (Academic Press, 2011).

24 Ueno, K., Inaba, A., Kondoh, M. \& Watanabe, M. Colloidal stability of bare and polymer-grafted silica nanoparticles in ionic liquids. Langmuir 24, 5253-5259 (2008).

25 Lin, M. Y., Lindsay, H. M., Weitz, D. A., Ball, R. C., Klein, R. \& Meakin, P. Universality in colloid aggregation. Nature 339, 360-362 (1989).

26 Ueno, K., Hata, K., Katakabe, T., Kondoh, M. \& Watanabe, M. Nanocomposite ion gels based on silica nanoparticles and an ionic liquid: ionic transport, viscoelastic properties, and microstructure. J. Phys. Chem. B 112, 9013-9019 (2008).

27 Khan, S. A. \& Zoeller, N. J. Dynamic rheological behavior of flocculated fumed silica suspensions. J. Rheol. 37, 1225-1235 (1993). 
28 Ueno, K., Imaizumi, S., Hata, K. \& Watanabe, M. Colloidal interaction in ionic liquids: effects of ionic structures and surface chemistry on rheology of silica colloidal dispersions. Langmuir 25, 825-831 (2009).

29 Ueno, K., Kasuya, M., Watanabe, M., Mizukami, M. \& Kurihara, K. Resonance shear measurement of nanoconfined ionic liquids. Phys. Chem. Chem. Phys. 12, 4066-4071 (2010).

30 Israelachvili, J. N. in Intermolecular and Surface Forces (Third Edition) 341-380 (Academic Press, 2011).

31 Hayes, R., Warr, G. G. \& Atkin, R. Structure and nanostructure in ionic liquids. Chem. Rev. 115, 6357-6426 (2015).

32 Hayes, R., Warr, G. G. \& Atkin, R. At the interface: solvation and designing ionic liquids. Physical Chemistry Chemical Physics 12, 1709-1723 (2010).

33 Smith, J. A., Werzer, O., Webber, G. B., Warr, G. G. \& Atkin, R. Surprising Particle Stability and Rapid Sedimentation Rates in an Ionic Liquid. J. Phys. Chem. Lett. 1, 64-68 (2010).

34 Gao, J., Ndong, R. S., Shiflett, M. B. \& Wagner, N. J. Creating nanoparticle stability in ionic liquid $\left[\mathrm{C}_{4} \mathrm{mim}\right]\left[\mathrm{BF}_{4}\right]$ by inducing solvation layering. ACS Nano 9, 3243-3253 (2015).

35 Zhang, H., Dasbiswas, K., Ludwig, N. B., Han, G., Lee, B., Vaikuntanathan, S., Talapin, D. V. Stable colloids in molten inorganic salts. Nature 542, 328 (2017).

36 Israelachvili, J. N. in Intermolecular and Surface Forces (Third Edition) 133-149 (Academic Press, 2011).

37 Ueno, K., Sano, Y., Inaba, A., Kondoh, M. \& Watanabe, M. Soft glassy colloidal arrays in an ionic liquid: colloidal glass transition, ionic transport, and structural color in relation to microstructure. J. Phys. Chem. B 114, 13095-13103 (2010).

38 Ueno, K., Fukai, T., Nagatsuka, T., Yasuda, T. \& Watanabe, M. Solubility of poly(methyl methacrylate) in ionic liquids in relation to solvent parameters. Langmuir 30, 3228-3235 (2014).

39 Batista, M. L. S., Neves, C. M. S. S., Carvalho, P. J., Gani, R. \& Coutinho, J. A. P. Chameleonic behavior of ionic liquids and its impact on the estimation of solubility parameters. J. Phys. Chem. $B$ 115, 12879-12888 (2011).

40 Huang, Y., Takata, A., Tsujii, Y. \& Ohno, K. Semisoft colloidal crystals in ionic liquids. Langmuir 33, 7130-7136 (2017).

41 Unemoto, A., Matsuo, T., Ogawa, H., Gambe, Y. \& Honma, I. Development of all-solid-state lithium battery using quasi-solidified tetraglyme-lithium bis(trifluoromethanesulfonyl)amidefumed silica nano-composites as electrolytes. J. Power Sources 244, 354-362 (2013). 
42 Chen, N., Zhang, H., Li, L., Chen, R. \& Guo, S. Ionogel electrolytes for high - performance lithium batteries: A review. Adv. Energy Maer., 1702675 (2018).

43 Ueki, T. Stimuli-responsive polymers in ionic liquids. Polym. J. 46, 646-655 (2014).

44 Ueno, K., Inaba, A., Ueki, T., Kondoh, M. \& Watanabe, M. Thermosensitive, soft glassy and structural colored colloidal array in ionic liquid: colloidal glass to gel transition. Langmuir 26, 18031-18038 (2010).

45 Ueno, K., Inaba, A., Sano, Y., Kondoh, M. \& Watanabe, M. A soft glassy colloidal array in ionic liquid, which exhibits homogeneous, non-brilliant and angle-independent structural colours. Chem. Commun., 3603-3605 (2009).

46 Jin, C., Meng, X., Cheng, B., Li, Z. \& Zhang, D. Photonic gap in amorphous photonic materials. Phys. Rev. B 63, 195107 (2001).

47 Ueno, K., Fukai, T. \& Watanabe, M. Thermosensitive soft glassy colloidal arrays of blockcopolymer-grafted silica nanoparticles in an ionic liquid. Polym. J. 48, 289-294 (2015). 